\title{
Suppression of a Prolyl 4 Hydroxylase Results in Delayed Abscission of Overripe Tomato Fruits
} Andreas Perrakis, Craita E. Bita, Stilianos Arhondakis, Afrodite Krokida, Khansa Mekkaoui,
Dusan Denic, Konstantinos N. Blazakis, Dimitrios Kaloudas and Panagiotis Kalaitzis*

Department of Horticultural Genetics and Biotechnology, Mediterranean Agronomic Institute of Chania (MAICh), Chania, Greece

The tomato pedicel abscission zone (AZ) is considered a model system for flower and fruit abscission development, activation, and progression. O-glycosylated proteins such as the Arabidopsis IDA (INFLORESCENCE DEFICIENT IN ABSCISSION) peptide and Arabinogalactan proteins (AGPs) which undergo proline hydroxylation were demonstrated

OPEN ACCESS

Edited by:

Dominique Van Der Straeten, Ghent University, Belgium

Reviewed by: Chunlin Shi,

University of Oslo, Norway Azeddine Driouich, Université de Rouen, France

${ }^{*}$ Correspondence: Panagiotis Kalaitzis panagiot@maich.gr

Specialty section: This article was submitted to Plant Physiology, a section of the journal

Frontiers in Plant Science

Received: 28 September 2018 Accepted: 07 March 2019

Published: 28 March 2019

Citation:

Perrakis A, Bita CE, Arhondakis S, Krokida A, Mekkaoui K, Denic D,

Blazakis KN, Kaloudas D and Kalaitzis $P$ (2019) Suppression of a Prolyl 4 Hydroxylase Results in Delayed Abscission of Overripe Tomato Fruits.

Front. Plant Sci. 10:348. doi: 10.3389/fp/s.2019.00348 to participate in abscission regulation. Considering that the frequency of occurrence of proline hydroxylation might determine the structure as well the function of such proteins, the expression of a tomato prolyl 4 hydroxylase, SIP4H3 (Solanum lycopersicum Prolyl 4 Hydroxylase 3) was suppressed in order to investigate the physiological significance of this post-translational modification in tomato abscission. Silencing of SIP4H3 resulted in the delay of abscission progression in overripe tomato fruits 90 days after the breaker stage. The cause of this delay was attributed to the downregulation of the expression of cell wall hydrolases such as SITAPGs (tomato abscission polygalacturonases) and cellulases as well as expansins. In addition, minor changes were observed in the mRNA levels of two SIAGPs and one extensin. Moreover, structural changes were observed in the silenced SIP4H3AZs. The fracture plane of the AZ was curved and not along a line as in wild type and there was a lack of lignin deposition in the AZs of overripe fruits 30 days after breaker. These results suggest that proline hydroxylation might play a role in the regulation of tomato pedicel abscission.

Keywords: tomato, prolyl-4-hydroxylase, abscission, overripe fruit, Arabinogalactan proteins, cell-wall

\section{INTRODUCTION}

Abscission is the process of organ separation in plants with significant implications in crop productivity considering that fruit and flower abscission might affect yield in several crops (Addicott, 1982; Tranbarger et al., 2017). Some plant species such as Arabidopsis and tomato are considered model systems for abscission studies, particularly Arabidopsis for flower abscission and tomato for pedicel abscission.

There are several advantages to study abscission in tomato considering the high-quality annotation of the tomato genome, updated and detailed transcriptome databases such as 
Tomexpress ${ }^{1}$ and tomato functional genomics database ${ }^{2}$, a tomato abscission zone-specific microarray (Meir et al., 2010; Sundaresan et al., 2016), abscission mutants such as the jointless (j) locus which lack the abscission zone (AZ) (Mao et al., 2000), and well-established transformation and, recently, genome editing methodologies. The AZ-specific microarray was constructed after RNAseq of samples from various developmental stages of leaf and flower AZs which resulted in the identification of 8,823 transcripts (Sundaresan et al., 2016).

The tomato pedicel comprises a distinct $\mathrm{AZ}$ at a predetermined position which is responsible for the detachment of either the flower or the fruit when the abscission process initiates. This AZ is comprised of six to eight cell layers at the open flower stage. At the overripe fruit stage, the AZs showed lignin deposition (Iwai et al., 2013; Tsuchiya et al., 2015) while no cellulose synthase, xyloglycan endotransglycosylase/hydrolase, and expansin signals were observed (Tsuchiya et al., 2015). A group of the expansin gene family comprising 16 members showed differential expression patterns in tomato leaf and flower AZs while most of them were mainly expressed in tomato leaf AZs (Sundaresan et al., 2016). Two of them, SlEXP1 and SlEXP11 were shown to be upregulated in flower AZs after $6 \mathrm{~h}$ of ethylene treatment (Wang et al., 2013).

Transcriptome analysis revealed that several key regulators of meristem-associated functions such as a tomato homolog of WUSCHEL (LeWUS), GOBLET (GOB), LATERAL SUPPRESSOR $(L s)$, and Blind $(B l)$ are specifically expressed in the pedicel AZ (Nakano and Ito, 2013). Among the transcription factors which are expressed in pedicel AZ, there is a member of the ERF (ethylene response family), the SlERF52 which was reported to play a regulatory role in the induction of the cell wall hydrolases during abscission progression (Nakano et al., 2014). Downregulation of SIRF52 delayed pedicel abscission due to the lack of upregulation of tomato abscission PGs (TAPGs) and cellulases (Nakano et al., 2014).

In Arabidopsis, the hormone peptide IDA (inflorescence deficient in abscission) and their interacting receptor-like kinases HAESA and HAESA-like2 are considered master regulators of flower abscission (Butenko et al., 2003; Stenvik et al., 2008). The Arabidopsis IDA peptide is hydroxylated at a proline residue in position 7 and this hydroxylation event increased the activity of the peptide by 30 -fold, indicating the functional significance of proline hydroxylation for IDA (Butenko and Simon, 2014). Five IDA genes were identified in tomato and only one of them, SIIDA1, was expressed in the leaf AZ as well as the petiole (Tucker and Yang, 2012). However, gene expression data alone could not lead to any conclusion related to the role of IDA in tomato abscission (Tucker and Yang, 2012). Moreover, there is no information whether the tomato IDA peptides are proline hydroxylated and the physiological importance of this hydroxylation. There are also 15 CLAVATE3/EMBRYOSURROUNDING REGION (CLE)-secreted peptide genes

${ }^{1}$ http://tomexpress.toulouse.inra.fr/

${ }^{2}$ http://ted.bti.cornell.edu/ which exhibited tissue- and organ-specific expression patterns while strong expression was observed during fruit development and ripening (Zhang et al., 2014). However, it has not been reported whether any of them is expressed in fruit or leaf AZs.

There are 10 tomato prolyl 4 hydroxylases (P4Hs) which were shown to be involved in cell division and expansion of leaves by using a virus-induced gene silencing (VIGS) approach (Fragkostefanakis et al., 2014). These phenotypes might be related to observed alterations in the protein content of substrate proteins such as Arabinogalactan proteins (AGPs) and extensins (Fragkostefanakis et al., 2012, 2014). Moreover, the use of 2-oxoglutarate analog pyridine 2,4-dicarboxylate resulted in a decrease in hydroxyproline content and in epitope bound AGPs according to western blot analysis (Fragkostefanakis et al., 2018). In addition, three Arabidopsis $\mathrm{P} 4 \mathrm{Hs}$ were reported to have a regulatory role in the root hair elongation process (Velasquez et al., 2011, 2015). The AtP4H2, AtP4H5, and AtP4H13 T-DNA knock out mutants exhibited a short root hair phenotype supporting the model that proline hydroxylation of hydroxyproline-rich glycoproteins and particularly extensins is important for cell wall assembly and function (Velasquez et al., 2011, 2015).

In this study, we report that suppression of SIP $4 \mathrm{H} 3$ expression resulted in the delay of pedicel abscission in overripe tomato fruits. This delay was attributed to suppression of expression of abscission-specific cell wall hydrolases.

\section{MATERIALS AND METHODS}

\section{Construction of Binary Vector, Agro-Mediated Transformation and Transgenic Plants}

The RNAi-SlP4H3 construct was generated by the amplification of a 838 bp fragment corresponding to base 168-1006 of the cDNA SIP4H3 gene (Solyc02g083390.2) using the SIP4H3 primers (Supplementary Table 1), containing attb sites and subcloned into the pDNR221 (accession number AF485783) entry vector (Invitrogen). The construct was recombined into the pHellsgate12 vector (a.n. AF489904) (CSIRO, Australia), using Gateway cloning technology (Invitrogen, Carlsbad, CA). The insertion in the binary vector was verified by sequencing, and used to transform tomato cotyledons Ailsa Craig (AC) by Agrobacterium tumefaciens (strain LBA4404) according to Fillatti et al. (1987) with some modifications. For positive control, an empty vector (pBi121, a.n. AF485783) was inserted into A. tumefaciens (strain LBA4404) and used for transformation of Ailsa Craig cotyledons. Transgenic plants were detected with PCR using the gene-specific primers for nptII cDNA. Nine independent transgenic lines as well as the empty vector (pBi121) were grown in parallel with an open hydroponic system in two separate net chambers in the experimental greenhouse of M.A.I.Ch. Tissues of AZs of overripe fruit 30 and 90 days after breaker from RNAi and wild type plants were collected, frozen in liquid nitrogen, and stored at $-80^{\circ} \mathrm{C}$ (Alba et al., 2005). 
Approximately $5 \mu \mathrm{g}$ of genomic DNA was extracted from transgenic and wild type plants as described by Sambrook et al. (1982) with few modifications, in order to confirm construct integration into the genome. PCR-specific primers of $n p t I I$ and SlP4H3 cDNAs were designed (Supplementary Table 1) and used to amplify a partial fragment of $n p t I I$ and SIP4H3. In brief, for each reaction, $50 \mathrm{ng}$ of template DNA, $1 \mathrm{X}$ buffer, $200 \mu \mathrm{M}$ dNTPs, $1.0 \mu \mathrm{M}$ of each primer, and 1.25 units Taq Polymerase (Invitrogen, ThermoFisher Scientific, Walttham, MA USA) were used in a final volume of $20 \mu \mathrm{l}$. Thermal conditions were $2 \mathrm{~min}$ at $96^{\circ} \mathrm{C}$ for denaturation, followed by 35 cycles of $1 \mathrm{~min}$ at $96^{\circ} \mathrm{C}$ for denaturation, $1 \mathrm{~min}$ at $55^{\circ} \mathrm{C}$ for annealing, and $2 \mathrm{~min}$ at $72^{\circ} \mathrm{C}$ for extension, followed by the last step at $72 \mathrm{C}$ for $10 \mathrm{~min}$. For negative control, distilled water was used. Amplification products were loaded in a $1.5 \%$ agarose gel with $1 x$ TBE running buffer, using a $1 \mathrm{~kb}$ DNA ladder (GeneRuler, Fermentas Life Sciences) and stained with GelRed (Biotium).

\section{RNA Extraction and cDNA Synthesis}

Total RNA was isolated from $200 \mathrm{mg}$ of tissue of $\mathrm{Br}+30$ and $\mathrm{Br}+90$ fruit $\mathrm{AZs}$ as well as leaf $\mathrm{AZs}$, from an empty vector (pBi121) line and three SIP4H3 RNAi lines (\#1, \#6, and \#7). Tissue was grounded in liquid nitrogen and total RNA was extracted by using the $\mathrm{RNeasy}^{\circledR}$ plant mini kit (QIAGEN) and PureLink ${ }^{\mathrm{TM}}$ RNA mini kit (ThermoFisher, Walttham, MA, USA). The DNAseI-RNAse free enzyme (ThermoFisher Scientific, Walttham, MA, USA) was used to remove the genomic DNA. Approximately $1 \mu \mathrm{g}$ of total RNA was reverse transcribed using SuperscriptII ${ }^{\circledR}$ Reverse Transcriptase (ThermoFisher Scientific, Walttham, MA, USA) and cDNA synthesis was performed following manufacturer's instructions using oligod $\mathrm{T}_{12-18}$ primers.

\section{qRT-PCR Analysis}

Gene expression analysis of selected genes was performed using a 48-well StepOnePlus ${ }^{\mathrm{TM}}$ Real-Time PCR System (ThermoFisher Scientific, Walttham, MA, USA) on three independent biological replicates. cDNA samples were prepared as described above and normalized using actin-specific primers (Supplementary Table 1). Standard dilution curves were performed for each PCR amplicon, and all data were normalized at the level of SlACTIN transcript. Primers were designed using the Primer Express v2.0 software (Applied Biosystems, Foster City, Calif) (Supplementary Table 1). The qRT-PCR reaction $(20 \mu \mathrm{l})$ mix consisted of gene-specific primers, as shown in Supplementary Table 1, SYBR ${ }^{\mathrm{TM}}$ Select Master Mix (ThermoFisher Scientific, Walttham, MA, USA), cDNA templates, and were run in StepOne ${ }^{\mathrm{TM}}$ Real-Time PCR system. The thermal cycling conditions were $50^{\circ} \mathrm{C}$ for $2 \mathrm{~min}$, $95^{\circ} \mathrm{C}$ for $10 \mathrm{~min}$ followed by $95^{\circ} \mathrm{C}$ for $15 \mathrm{~s}, 60^{\circ} \mathrm{C}$ for $30 \mathrm{~s}$, and $72^{\circ} \mathrm{C}$ for $30 \mathrm{~s}$ for 40 cycles. Data were analyzed using the $2^{-\Delta \Delta C T}$ method (Livak and Schmittgen, 2001) and presented as relative levels of gene expression. Standard errors were calculated for all mean values.

\section{Statistical Analysis}

The numerical data were analyzed and post-processed using the statistics toolbox of MATLAB (The Mathworks Inc., Natick, MA, USA). The standard descriptive statistical methods were applied on the numerical findings and the data re-expressed as mean \pm standard errors (SEs). Then one-way analysis of variance (one-way ANOVA) was applied on the data of each line, to analyze and determine possible statistically significant differences in the data sets. Furthermore, we used a post hoc multicomparison approach (Tukey's honest significant difference criterion, 95\% confidence interval) to identify the differences between wild type (WT) and each RNAi line.

\section{LIGNIN Staining With Phloroglucinol-HCI}

Approximately $0.3 \mathrm{~g}$ of phloroglucinol was dissolved in $10 \mathrm{ml}$ of absolute ethanol to prepare a $3 \%$ phloroglucinol solution. One volume of concentrated $\mathrm{HCl}(37 \mathrm{~N})$ was mixed with two volumes of $3 \%$ phloroglucinol in ethanol to prepare the phloroglucinol- $\mathrm{HCl}(\mathrm{Ph}-\mathrm{HCl})$. The $\mathrm{AZ}$ sections were transferred to a $2.0-\mathrm{ml}$ microcentrifuge tube and $1 \mathrm{ml}$ of $\mathrm{Ph}-\mathrm{HCl}$ solution was added to the tube. The tube was shaken gently to assure that all the sections are stained. The observation was made under bright-field lighting microscope.

\section{Plant Material and Flower Abscission Zone Sections}

The tomato plants were grown in the greenhouse of the Mediterranean Agronomic Institute of Chania under regular conditions. The leaf abscission explants were prepared from stems of mature tomato plants comprising around $10 \mathrm{~cm}$ of stem section with the petiole after removing the leaf blade. The explants were then placed upright in beakers with water and treated with approximately $15 \mu \mathrm{L}^{-1}$ of ethylene in jars with an open system installation for $48 \mathrm{~h}$. The flower AZs were fixed in $10 \%$ formalin, then dehydrated through a series of graded ethanol and embedded in paraffin by using a paraffin embedding station (Leica TP1020). The AZs were then used to prepare longitudinal sections with a rotary microtome (Leica RM2135) for image analysis with a stereomicroscope Leica MZ7.5 (Meyer Instruments Inc.), a camera ProgRes ${ }^{\circledR} \mathrm{C} 12$ plus (Jenoptik), and the CapturePro ${ }^{\circledR} 2.1$ Image Acquisition Software.

\section{RESULTS}

\section{Suppression of SIP4H3 by Using an RNAi Approach}

Ten $\mathrm{P} 4 \mathrm{H}$ genes were identified in the S. lycopersicum genome while a dendrogram which was prepared after comparison of their deduced amino acid sequences indicated clustering into three groups (Fragkostefanakis et al., 2014). Among them, 3 tomato P4Hs, SIP4H3, SIP4H8, and SIP4H10, and another 6 out of 13 Arabidopsis $\mathrm{P} 4 \mathrm{Hs}$ were grouped into the same subcluster of cluster A (Fragkostefanakis et al., 2014). This indicates higher homology of their deduced amino acid sequence to Arabidopsis $\mathrm{P} 4 \mathrm{Hs}$ compared to the other tomato $\mathrm{P} 4 \mathrm{Hs}$. Arabidopsis $\mathrm{P} 4 \mathrm{Hs}$ 
were reported to be involved with a regulatory role in growth programs such as root hair elongation (Velasquez et al., 2011, 2015). Moreover, silencing of additional tomato P4Hs by using stable transformation and transient approaches such as VIGS indicated that $\mathrm{SIP} 4 \mathrm{H} 3$ exhibited interesting phenotypes compared to other $\mathrm{P} 4 \mathrm{Hs}$ (data not shown). Therefore, among the three, the tomato SIP4H3 was initially selected for further investigation.

A specific RNAi silencing construct for SIP4H3 whose expression was directed by the $35 \mathrm{~S}$ promoter was transformed into wild type (Ailsa Craig) tomato plants by using A. tumefaciens-mediated transformation and nine independent transformants were generated. The construct integration was confirmed by the presence of $n p t I I$ in each line and three lines, RNAi\#1, RNAi\#6, and RNAi\#7, were further characterized at the T2 generation. The expression of SlP4H3 was determined at various tissues such as leaf, root, seed, and overripe fruit by qPCR (Figure 1A). The higher expression was determined in root and leaf while lower levels of expression were determined in the seed and overripe fruit (Figure 1A). Moreover, the levels of suppression of SIP4H3 were determined in the same tissues in all three lines, RNAi\#1, RNAi\#6, and RNAi\#7 (Figure 1B). Significant suppression was observed in all tissues with the highest decrease in expression detected in leaf tissue among the three RNAi lines (Figure 1B). Moreover, the SlP4H3 RNAi line \#1 showed the highest percentage of suppression in root and seed tissue (Figure 1B).

\section{SIP4H3 RNAi Lines Exhibited a Delayed Abscission Phenotype}

Wild type overripe tomato fruits abscised naturally usually around 90 days after the breaker stage under normal growing conditions (Figure 2A). However, the overripe fruits of three SIP4H3 RNAi lines did not abscise 90 days after breaker as shown in Figure 2A. Even tomato fruits, which were in terms of development at the

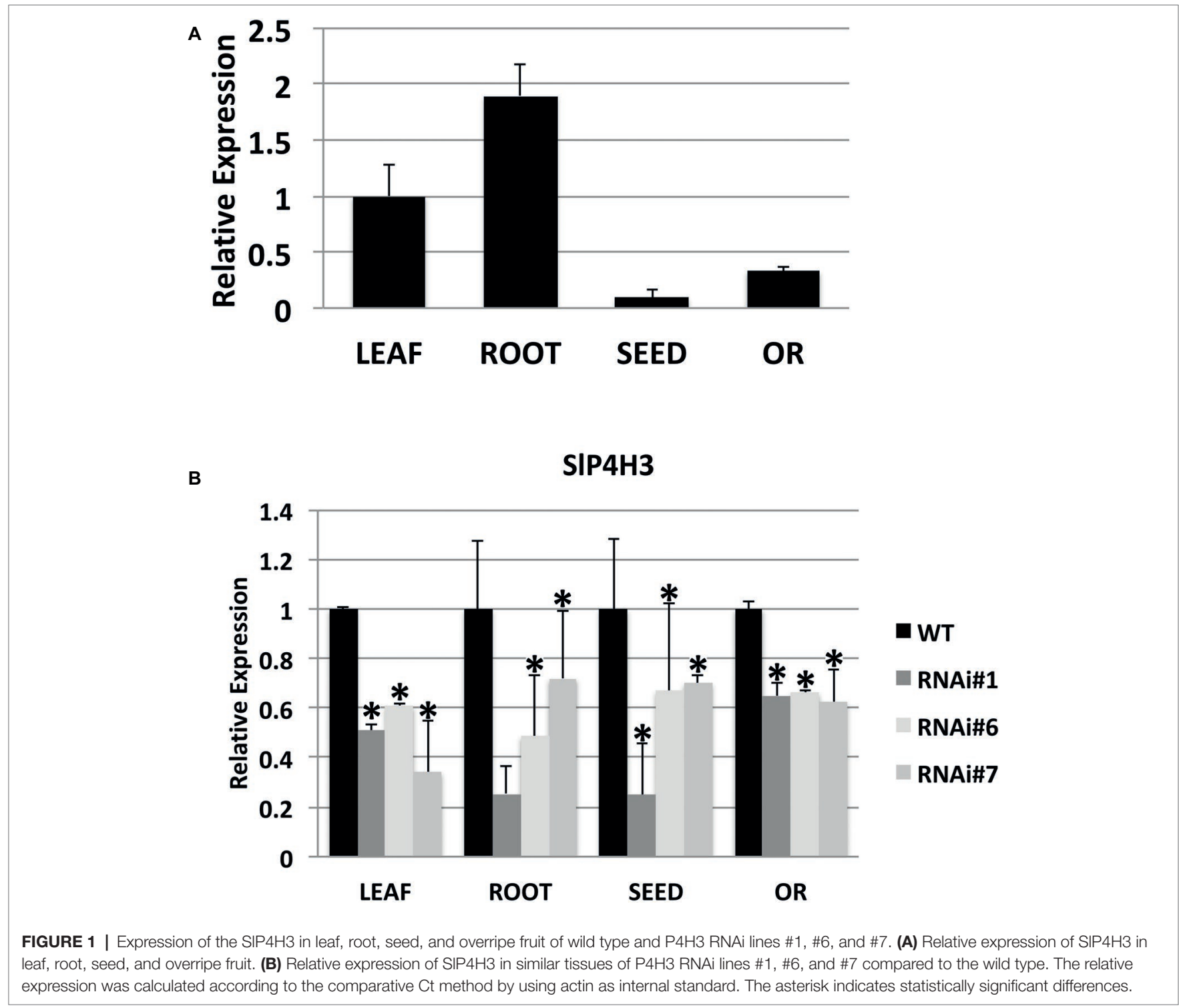


final stages of senescence, showed a delayed abscission phenotype (Figure 2A). This significant delay in fruit abscission was observed in all nine independent transformants. Moreover, the hydroxylation of specific proline residues is important for IDA activity, suggesting a putative role for this post-translational modification in abscission regulation in Arabidopsis (Butenko and Simon, 2014).

The specific repression of SIP4H3 in the fruit AZs of wild type and RNAi lines was verified by determining its expression levels. Total RNA was extracted from AZs of wild type and RNAi lines \#1, \#6, and \#7 fruits 90 days after the breaker stage and used for RT-qPCR analysis (Figure 3). AZs of overripe fruits were used for SIP4H3 expression by using qPCR indicating that the expression levels were partially suppressed in all three lines compared to the wild type (Figure 3). The expression of the additional tomato $\mathrm{P} 4 \mathrm{Hs}$ was investigated in the fruit AZ 90 days after the breaker stage in order to determine whether their expression levels were altered in response to the silencing of SlP4H3 (Supplementary Figure 1). The transcript abundance of seven $\mathrm{P} 4 \mathrm{Hs}$ was detected, and among them SlP4H1, SLP4H2, and SIP4H5 exhibited similar to wild type expression levels (Supplementary Figure 1). However, only SIP4H9 showed significant upregulation in all three RNAi lines, while SIP4H4, SIP4H6, and SIP4H8 showed significant upregulation only in two and one RNAi lines, respectively (Supplementary Figure 1). No detectable expression of SIP4H7 was observed. These results indicate that no other tomato $\mathrm{P} 4 \mathrm{H}$ was downregulated in the SIP4H3 RNAi lines.

The AZ of fully open flowers is comprised of six to eight layers of small, disk-shaped cells with dense cytoplasm, which is considered a very distinct, specialized structure of the pedicel (Tabuchi et al., 2001). This structure was investigated in the SIP4H3 RNAi lines by longitudinal sections of the AZ of fully open flowers indicating similar anatomical alterations in all three RNAi lines (Figure 2B). The RNAi AZ specialized cells

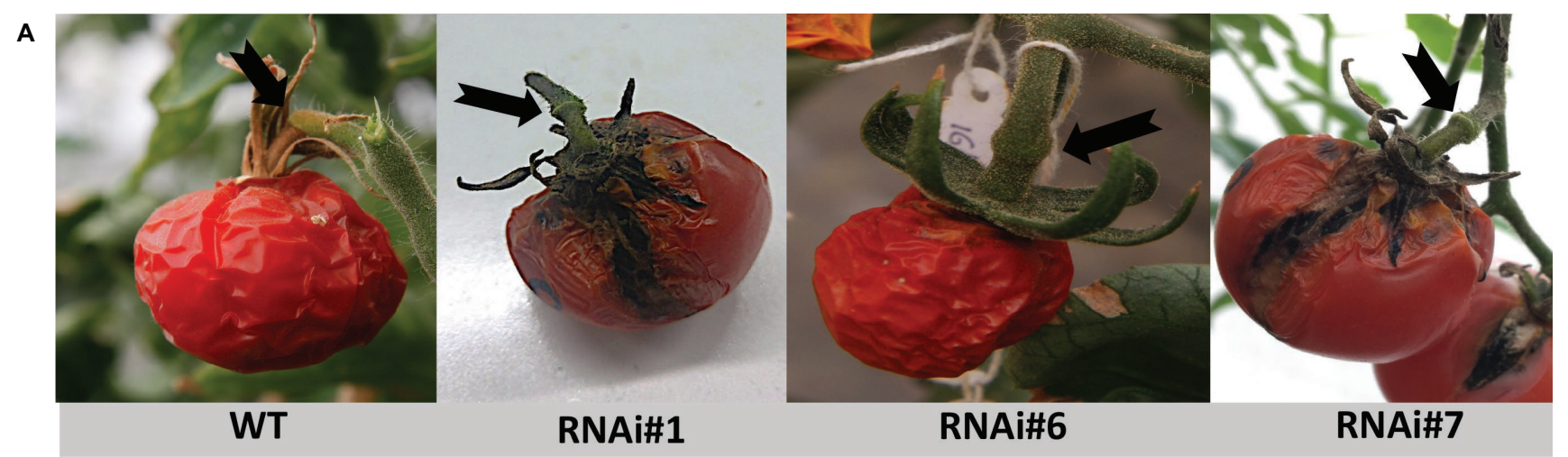

B
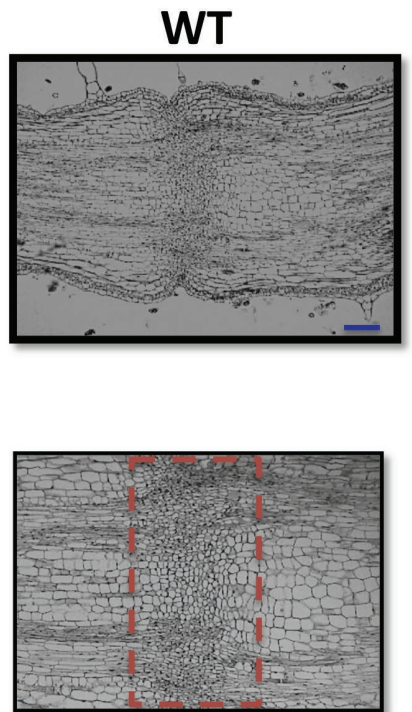

RNAi\#1
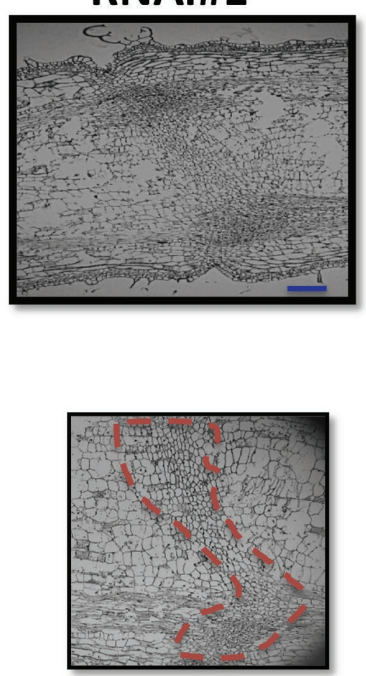

RNAi\#6
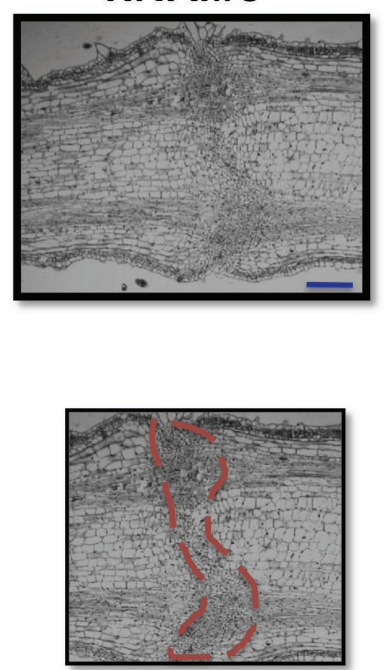

RNAi\#7
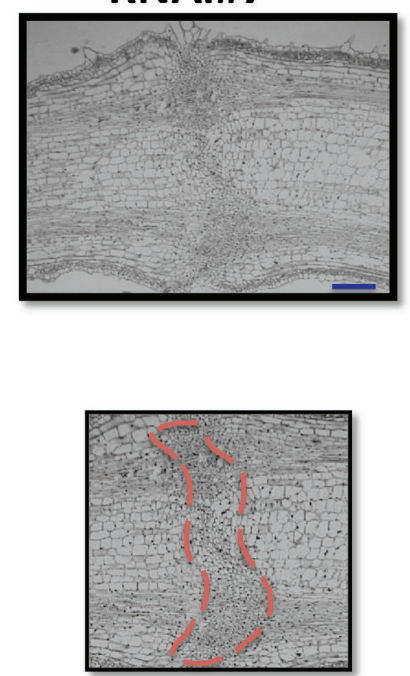

FIGURE 2 | Delay of overripe fruit abscission and morphological structure of AZs in SIP4H3 RNAi lines. (A) Final stage of abscission of a wild type overripe fruit 90 days after breaker. Representative AZs of overripe fruits 90 days after breaker in the three SIP4H3 RNAi lines \#1, \#6, and \#7. The arrows point to the AZ. (B) Longitudinal sections of petiole AZs at the stage of open flower in the SIP4H3 RNAi lines and wild type. The dotted-line contour defines the abscission zone based on the visible separation layer. The blue line represents $1 \mathrm{~mm}$. 


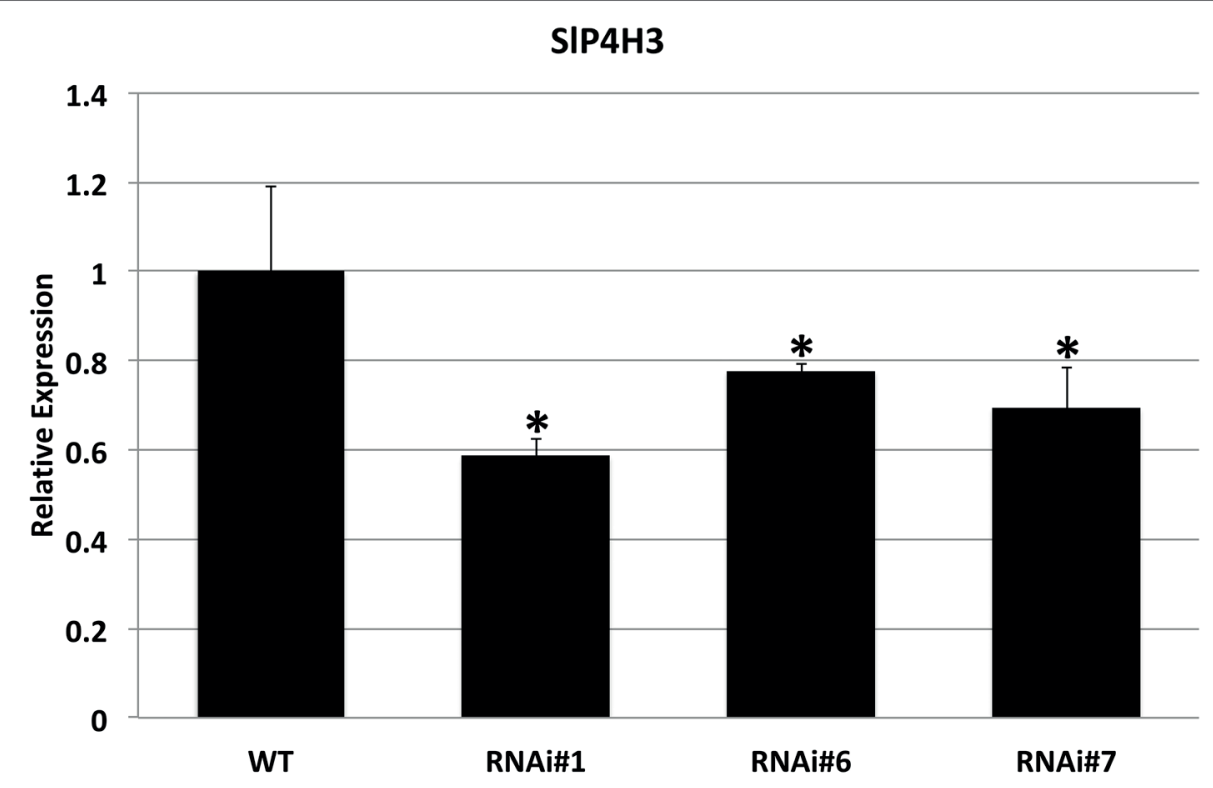

FIGURE 3 | Expression analysis of the SIP4H3 in the AZs of overripe fruits 90 days after breaker in the three SIP4H3 RNAi lines \#1, \#6, and \#7 and wild type (WT) plants. Relative expression of SIP4H3 transcript in three RNA lines and WT AZs. The relative expression was calculated according to the comparative Ct method by using actin as internal standard. The asterisk indicates statistically significant differences.

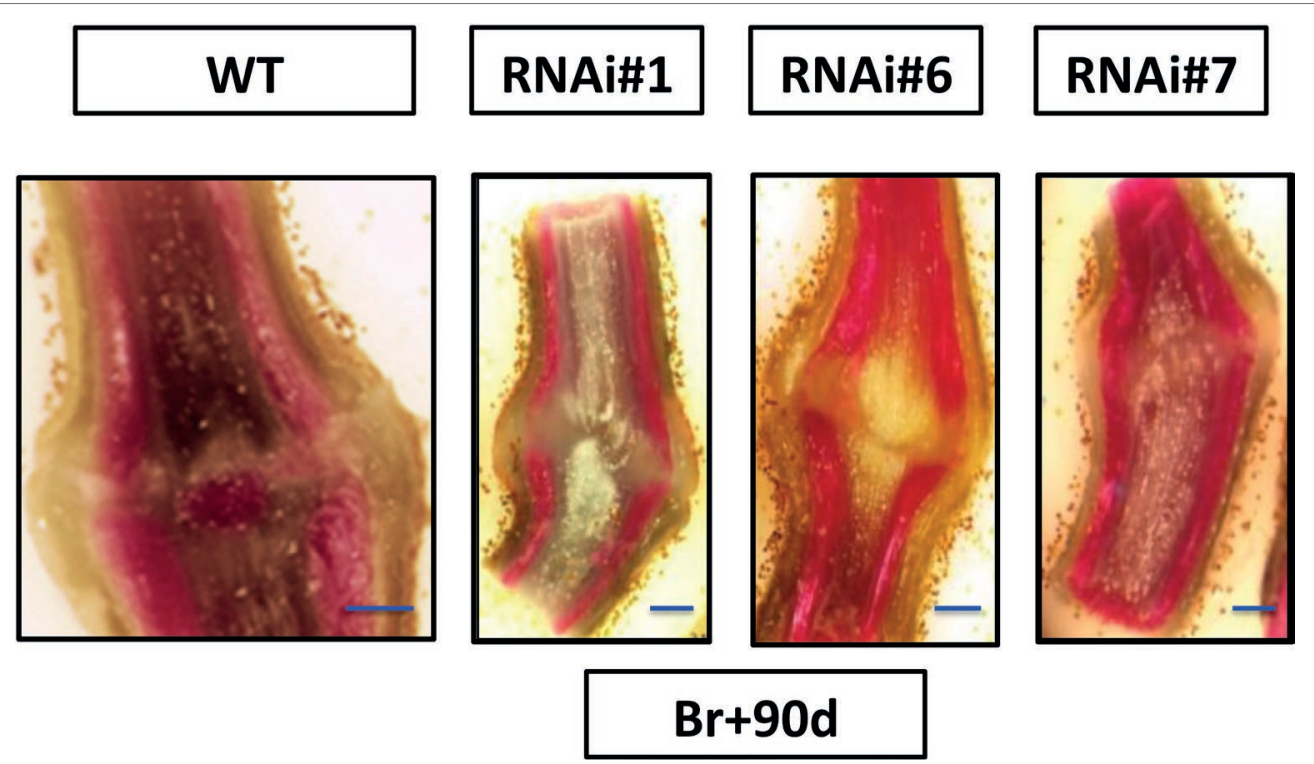

FIGURE 4 | Analysis of longitudinal sections of fruit AZs 90 days after breaker. Fruit AZs 90 days after breaker were stained for lignin deposition with phloroglucinol$\mathrm{HCl}$ in the three RNAi lines \#1, \#6, and \#7 and wild type. The curved fracture plane can be observed in the AZs of the RNAi lines compared to the wild type (WT). The blue line represents $1 \mathrm{~mm}$.

were not positioned along a straight line as in the wild type but the AZ plane was curved as clearly shown by the outline of the dotted lines (Figure 2B). Moreover, a variation in the number of $\mathrm{AZ}$ cell layers was observed with lower number of cell layers in the central parenchymatic region (Figure 2B).

Moreover, lignin deposition was observed in the AZ of the wild type sections at 90 days after breaker while no lignification was detected in the AZs of the RNAi lines' pedicel at 90 days after Breaker (Figure 4). These results might be related to the delay in abscission of the overripe fruits of SIP4H3 RNAi lines.

The SIP4H3 involvement in the progression of ethylene-induced petiole abscission was also investigated. Initially, the expression of SIP4H3 was determined in leaf AZ explants of the three RNAi lines and wild type (Figure 5A). Statistically significant downregulation was observed in all RNAi lines compared to wild type (Figure 5A). In addition, the leaf AZ explants were 


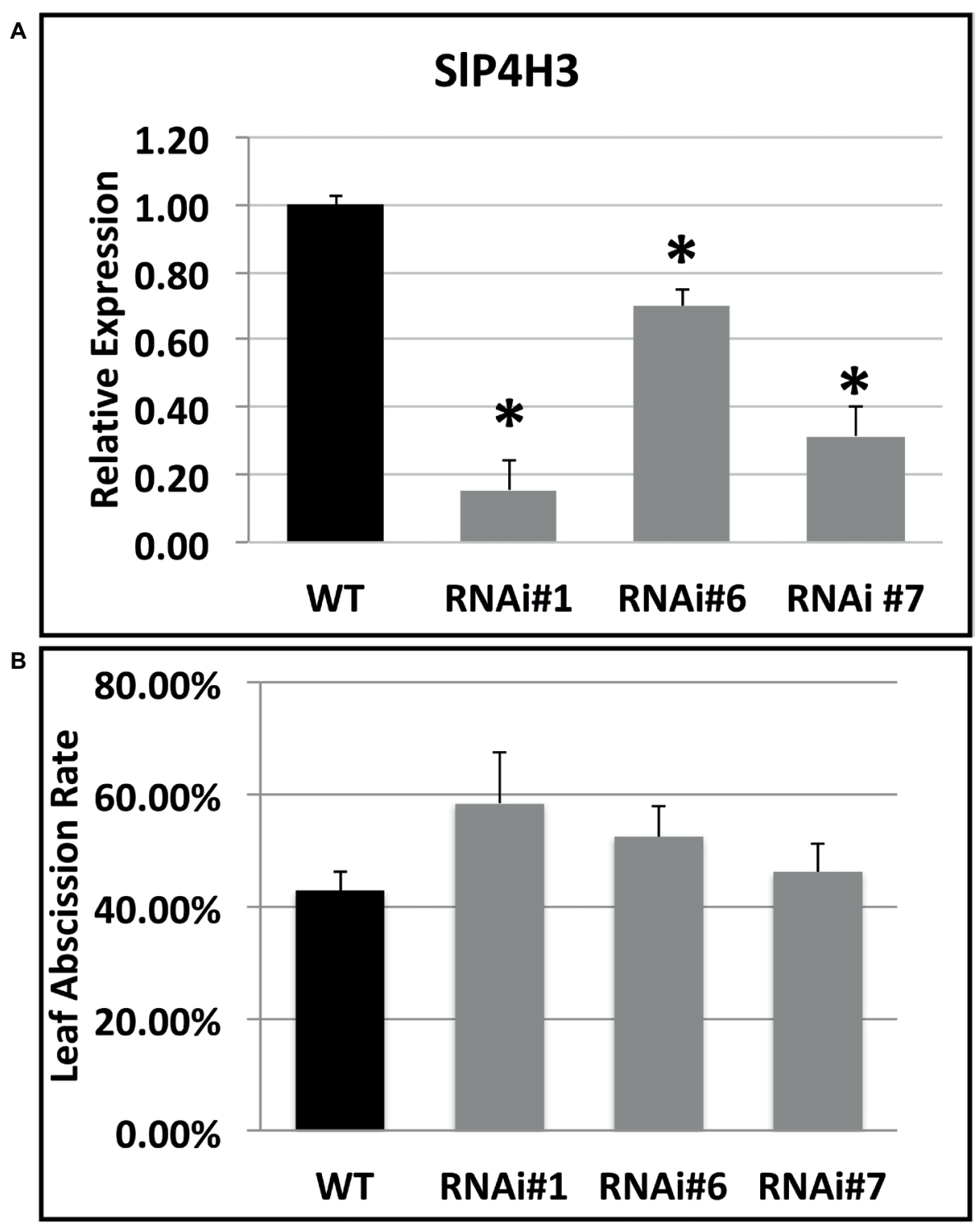

FIGURE 5 | Expression analysis of the SIP4H3 in petiole AZs and leaf abscission rate after 48 h of ethylene treatment. (A) Relative expression of SIP4H3 transcript in three RNA lines and WT petiole AZs. The relative expression was calculated according to the comparative Ct method by using actin as internal standard. The asterisk indicates statistically significant differences. (B) The petiole abscission kinetics expressed as percentages of pedicel abscission were determined after deblading the leaf explants and treating them with approximately $15 \mu \mathrm{L} \mathrm{L}^{-1}$ of ethylene in the three SIP4H3 RNAi lines \#1, \#6, and \#7 and the wild type. Three biological replicates were performed.

treated with $15 \mu \mathrm{l} \mathrm{L} \mathrm{L}^{-1}$ ethylene for $48 \mathrm{~h}$ in order to determine whether there are differences in the percentage of the abscission rate in response to ethylene. No statistically significant differences were observed among the three RNAi lines and wild type, indicating that ethylene-induced leaf abscission might not be affected by the levels of expression of SIP4H3 (Figure 5B).

\section{Gene Expression in Fruit Abscission Zones 90 Days After the Breaker Stage}

The expression of fruit abscission-specific genes was characterized in order to investigate the molecular basis of this delay in abscission. The patterns of expression of cell wall hydrolases such as SITAPG1, SITAPG2, SITAPG4, and SlCEL2, SlCEL5 as well as additional mRNAs such as SlEXP 1, SlEXP4, and SlERF52 were determined (Figure 6). SlEXP1 is considered a fruit ripening-regulated expansin with high levels of expression in tomato leaf AZs while SIEXP4 showed similar levels of transcript abundance in both leaf and flower AZs (Sundaresan et al., 2016). In addition, SIEXP1 was also shown to be upregulated in flower AZs after $6 \mathrm{~h}$ of ethylene treatment (Wang et al., 2013). Total RNA was extracted from RNAi lines $\# 1$, \#6, and \#7 fruit AZs at 90 days after the breaker stage and wild type and used for qPCR analysis.

The transcript levels of the five cell wall hydrolases, SITAPG1, SITAPG2, SITAPG4, and SICEL2, SICEL5, were determined in 


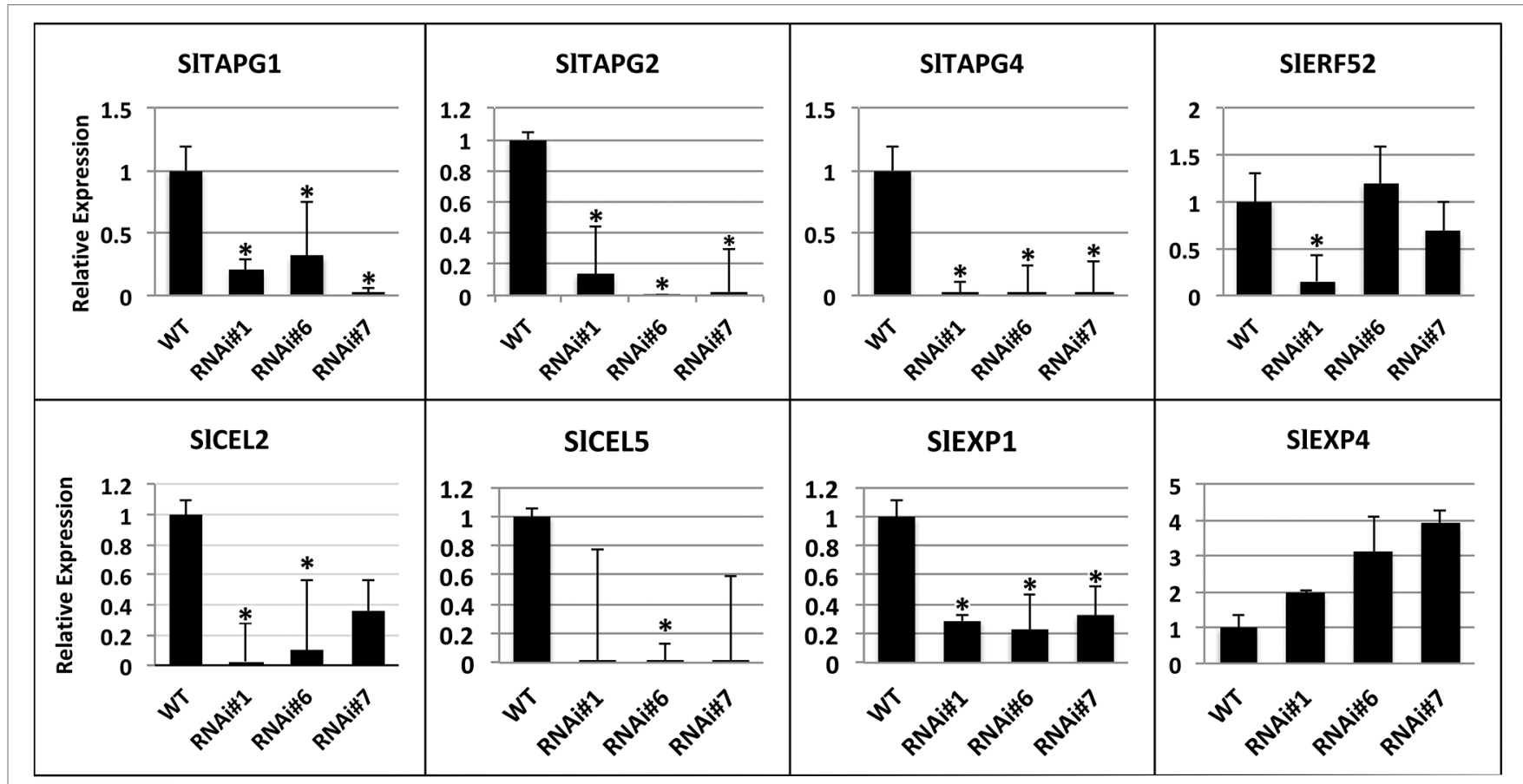

FIGURE 6 | Expression analysis of SITAPG1 (polygalacturonase), SITAPG2, SITAPG4, SICEL2, (cellulase), SICEL5, and SIEXP1 (expansin 1), SIEXP4, SIERF52 genes in AZs of SIP4H3 RNAi lines \#1, \#6, and \#7 and wild type (WT). The relative expression was calculated according to the comparative Ct method by using actin as internal standard. The asterisk indicates statistically significant differences.
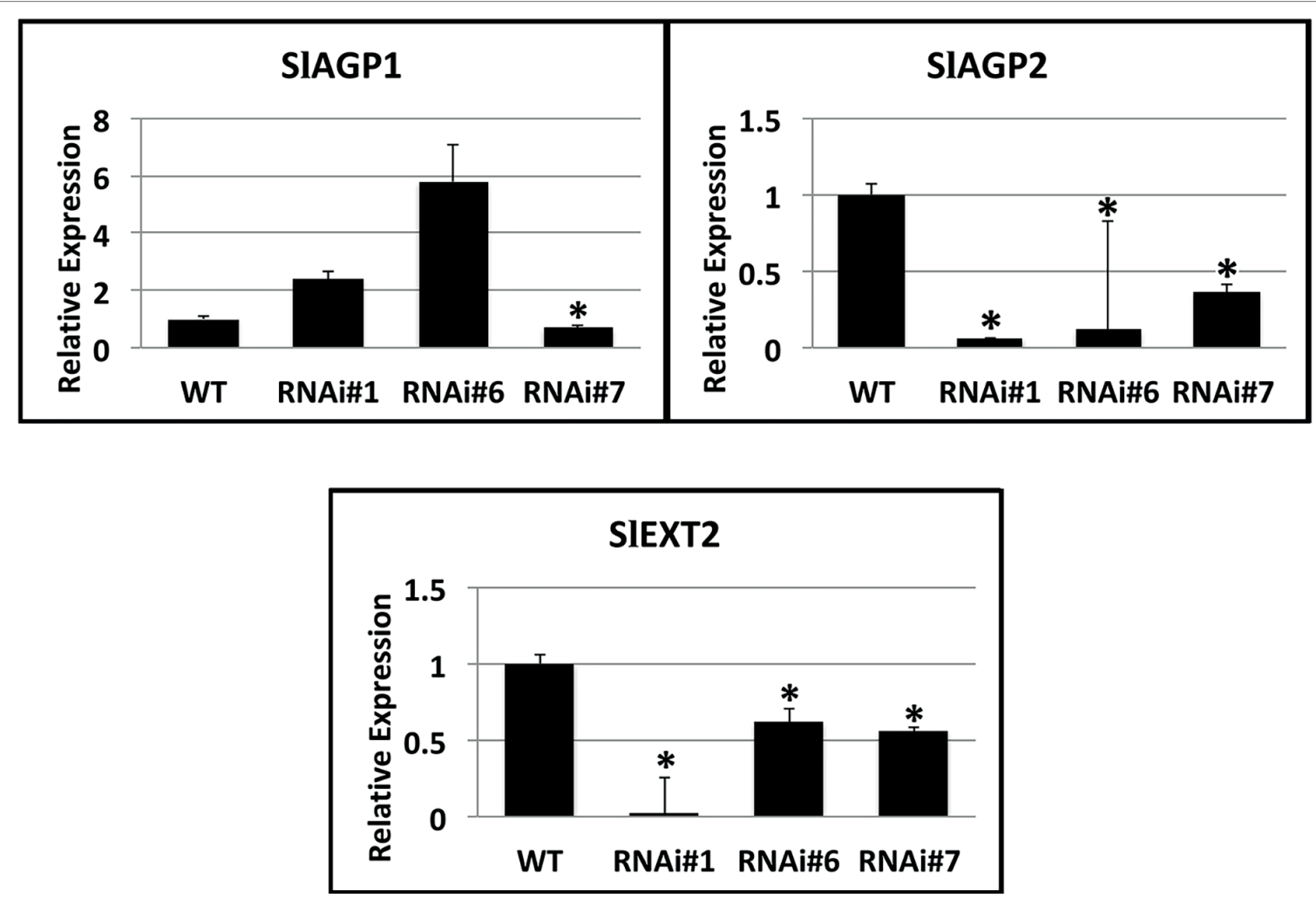

FIGURE 7 | Expression analysis of SIAGP1 (arabinogalactan protein 1) (Solyc02g092790.2.1), SIAGP2 (Solyc07g053640.1), and SIEXT2 (extensin 2) (solyc09g098510.3) genes in AZs of SIP4H3 RNAi lines \#1, \#6, and \#7 and wild type (WT). The relative expression was calculated according to the comparative Ct method by using actin as internal standard. The asterisk indicates statistically significant differences. 
the overripe AZs considering the high levels of expression of SITAPG1, SITAPG2, and SITAPG4 in tomato flower AZs (Kalaitzis et al., 1997; Sundaresan et al., 2016) and of SlCEL5 and SICEL2 in both flower and leaf AZs (Sundaresan et al., 2016). The expression levels of SITAPG1, SITAPG2, SITAPG4, and SlEXP1 were significantly downregulated by approximately $80-90 \%$ in all three SlP4H3 lines, justifying the delay in overripe fruit abscission compared to the wild type (Figure 6). However, the expression of SICEL2 and SICEL5 decreased in the RNAi lines but was not statistically significant for RNAi line \#7 and RNAi lines \#1 and \#7, respectively (Figure 6). Moreover, the transcript levels of EXP4 were not altered significantly in the RNAi lines (Figure 6). The expression of SlERF52 was not altered significantly in RNAi lines \#6 and \#7, just downregulation in RNAi line \#1 was observed (Figure 6).

Considering that the substrate proteins for proline hydroxylation are AGPs and extensins, a search in the Affymetrix microarrays of SlP4H3 line \#6 and wild type fruit AZs at 30 and 90 days after the breaker stage for transcripts encoding these polypeptides resulted in the identification of two SIAGPs, SlAGP1 and SlAGP2, and one extensin, SlEXT2 (Figure 7). RT-qPCR analysis indicated that SlAGP2 and SlEXT2 showed significantly lower levels of expression compared to wild type (Figure 7). The SlAGP1 transcript showed similar levels in RNAi lines \#1 and \#6 and lower levels in line \#7 (Figure 7). These results indicate overall lower levels of SIAGPs and extensin transcript abundance in RNAi lines.

\section{DISCUSSION}

$\mathrm{P} 4 \mathrm{Hs}$ catalyze the post-translational modification of proline hydroxylation in HRGPs such as AGPs and extensins as well as in hormone peptides such as the Arabidopsis IDA and CLAVATA3 (Matsubayashi, 2014).

With regard to AGPs, it was observed that Arabinogalactan was secreted in the AZs of Arabidopsis flowers after staining with the $\beta$-Yariv reagent which is known to bind AGPs (Stenvik et al., 2006). The presence of AGPs by using the JIM13 antibody was detected only in the IDA overexpressing flower AZs and not in wild type or IDA knock out mutant (Stenvik et al., 2006). This expression of the JIM13-bound epitopes was attributed to the upregulation of the AGP24 transcript in the IDA overexpressing floral AZs (Stenvik et al., 2006) indicating that AGPs might have a physiological role in progression of flower abscission. This is in accordance to our results that two SlAGPs and one extensin are expressed in the overripe fruit AZs. However, lower levels of SlAGP2 and extensin 2 were observed in their expression patterns in the RNAi lines compared to wild type, despite the fact that SIP4Hs catalyze a post-translational modification. These results indicate that proline hydroxylation might be involved in the regulation of transcription factors' stability considering similar involvement in all metazoan species (Semenza, 2010). There are proline hydroxylation motifs which were identified in the polypeptide sequences of transcription factors induced by hypoxia in Arabidopsis (Vlad et al., 2007). In this case, alterations in the expression of $\mathrm{SlP} 4 \mathrm{Hs}$ might induce alterations in the activity of transcription factors resulting in changes in gene expression of a wide array of genes comprising also AGPs and extensins as shown in Figure 7.

However, no differences were observed for the expression of SlAGP1 in lines \#6 and \#7. This suggests that there might be alterations for SlAGP1 at post-transcriptional level and probably at their protein content as previously reported in tomato (Fragkostefanakis et al., 2014). VIGS-induced suppression of three tomato $\mathrm{P} 4 \mathrm{Hs}$ was accompanied by a decrease in protein content of AGPs and extensins in roots and shoots (Fragkostefanakis et al., 2014).

It was previously reported that RNAi-induced knock down of SlERF52 delayed pedicel abscission at the stage of anthesis and that the cause of this delay was attributed to the suppression of expression of SITAPGs and cellulases (Nakano et al., 2014). In the SlP4H3 RNAi lines, the expression of the SlERF52 was not consistent showing minor alterations in expression levels compared to the wild type for lines \#6 and \#7 and lower expression levels for line \#1 (Figure 6). These results suggest that SIERF52 might not be involved in the delay of overripe fruit abscission in the SlP4H3 RNAi lines considering that no significant changes in its expression were observed.

VIGS-induced suppression of SITAPG1 expression probably resulted in the silencing of all five PGs in the petiole AZs and therefore in the inhibition of tomato petiole abscission (Jiang et al., 2008). In the same report, the silencing of two expansins and two endoglucanases did not affect flower abscission (Jiang et al., 2008). Therefore, the significant downregulation of SITAPG1, SITAPG2, and SITAPG4 in the three SIP4H3 RNAi lines might be considered responsible for the delay in overripe fruit abscission of SIP4H3 suppression lines regardless of the levels of expression of endoglucanases and expansins (Figure 6). It is interesting to note that the regulation of a post-translational modification, such as proline hydroxylation, induces alterations in the expression levels of a plethora of genes with different functions. There is an example in which the stability of a transcriptional activator such as the hypoxiainducible factor $1 \alpha$ (HIF-1 $\alpha$ ) which is considered a global regulator of hypoxic response in mammalian systems is regulated by proline hydroxylation (Koyasu et al., 2017). Three prolyl 4 hydroxylases (PHDs) trigger the ubiquitin-dependent degradation of HIF-1 $\alpha$ and by this way regulate the stability of this transcription factor (Koyasu et al., 2017). However, no additional lines of evidence were reported up to now indicating proline hydroxylation of transcription factors in plants.

Moreover, a KNOTTED-LIKE HOMEOBOX (KD1) protein is also considered a regulator of tomato leaf and flower abscission (Ma et al., 2015). Decrease of KD1 mRNA levels resulted in significant retardation of petiole and pedicel abscission while overexpression of this gene in a semidominant mutant, Petroselinum, accelerated abscission (Ma et al., 2015). Although suppression of SlP4H3 transcript caused a delay of overripe fruit abscission as in KD1-silenced tomato plants, preliminary analysis of transgenic lines overexpressing SlP4H3 did not show any acceleration in overripe fruit abscission (data not shown). Moreover, the suppression of SlP4H3 delayed pedicel abscission but did not affect petiole abscission indicating that proline 
hydroxylation might be involved in a different abscission regulatory mechanism compared to $\mathrm{KD} 1$ or alternatively, other $\mathrm{P} 4 \mathrm{H}(\mathrm{s})$ might be involved in the regulation of leaf abscission in tomato. These results suggest that there might be differences between petiole and pedicel abscission at the molecular level in tomato, although global transcriptome analysis indicated similar regulation of flower and leaf abscission (Sundaresan et al., 2016). However, it should be noted that P4Hs catalyze a post-translational modification which is not related to transcriptome regulation.

Antibodies for immunolocalization of xyloglycan endotransglycosylase/hydrolases (XTH) and expansins in tomato flower and fruit AZs showed detection of both proteins in flower AZ but complete lack of expression in overripe fruit AZ (Tsuchiya et al., 2015). Intense labeling was observed only in the vascular bundles of overripe fruit pedicels (Tsuchiya et al., 2015). Expansin 1 (SlEXP1) was strongly expressed in AZs 90 days after breaker (Figure 6). Although these results are not in accordance with the report by Tsuchiya et al. (2015), it should be taken into consideration the different fruit developmental stages which were used for the immunolocalization of XTH and EXP and their gene expression patterns by $\mathrm{qPCR}$ analysis, respectively. The overripe fruit AZs used for the immunolocalization studies probably refer to fruit ripening stages just several days after the red ripe stage (Tsuchiya et al., 2015).

Significant structural differences were observed in the AZ of the RNAi lines compared to the wild type considering that the fracture plane was curved and not across a line (Tabuchi et al., 2001), indicating alterations in the structure and development of pedicel AZ. The tomato pedicel AZ is comprised of cells arrested in an undifferentiated stage (van Nocker, 2009) and the observed curved fracture plane suggests that the developmental program of differentiation of specific cells was not executed properly. Therefore, the expression of the genes involved in the development of the pedicel AZ in RNAi lines needs to be determined at the early stage of flower $A Z$ development (Ito and Nakano, 2015).

Moreover, extensive lignification was observed in the AZs of the overripe but not of mature green fruits (Tsuchiya et al., 2015). Similar lignin deposition was also detected in the AZs 90 days after breaker only in the wild type but not in the RNAi lines (Figure 4). This lignification was associated with abscission-specific activation (Tsuchiya et al., 2015) which is in accordance with the lack of lignification in the AZ of the RNAi lines 90 days after breaker considering the delay in abscission activation. According to the previous (Iwai et al., 2013; Tsuchiya et al., 2015) and this report, lignin deposition might be considered a marker of abscission activation. Strong lignin deposition was also observed in the vascular bundles of AZs at 90 days after breaker in the wild type and RNAi lines. Similar results were also reported in previous studies (Iwai et al., 2013; Tsuchiya et al., 2015).

The suppression of SIP4H3 expression resulted in the delay of the overripe tomato fruit abscission due to the significant downregulation of the abscission polygalacturonases and cellulases as well as an expansin. Moreover, alterations were also observed in the structure of the AZs the significance of which remains to be elucidated. These results suggest that proline hydroxylation might play a key role in the induction of abscission cell wall hydrolases indicating an additional level of regulation of tomato pedicel abscission. Therefore, further studies are under way to investigate the $\mathrm{AZ}$ of red ripe fruits in response to ethylene in the RNAi as well as overexpression lines.

\section{AUTHOR CONTRIBUTIONS}

PK conceived and designed the work. PK, AP, CB, SA, AP, $\mathrm{AK}, \mathrm{KM}, \mathrm{DD}, \mathrm{KB}$, and $\mathrm{DK}$ were involved in the experiments, analysis, and interpretation of the data. $\mathrm{PK}, \mathrm{AP}, \mathrm{CB}, \mathrm{SA}, \mathrm{AP}$, $\mathrm{AK}, \mathrm{KM}, \mathrm{DD}, \mathrm{KB}$, and $\mathrm{DK}$ were involved in drafting the work. All authors revised and approved the final version.

\section{FUNDING}

This work has been supported partly by the project "Tomato P4Hs" (Ref. Number 4388), which was implemented by "ARISTEIA II", an Action of the "Operational Programme Education and Life Long Learning," co-funded by the European Social Fund (ESG) and National Resources. Moreover, this research was partially supported by the project "PlantUp: Upgrading Plant Capital" (MIS 5002803) which is implemented under the Action "Reinforcement of the Research and Innovation Infrastructure," funded by the "Operational Programme, Competitveness, Entrepreneurship and Innovation" (NSRF 2014-2020) and co-financed by Greece and European Union (European Regional Development Fund).

\section{ACKNOWLEDGMENTS}

We would like to thank Mrs. Maria Kosma and Mrs. Aline Monzer and Mr. Theodoraq Spano for their excellent technical assistance.

\section{SUPPLEMENTARY MATERIAL}

The Supplementary Material for this article can be found online at: https://www.frontiersin.org/articles/10.3389/fpls.2019.00348/ full\#supplementary-material

\section{SUPPLEMENTARY FIGURE 1 | Expression analysis of SIP4H1} (solyc02g064750), SIP4H2 (Solyc06g054490), SIP4H4 (Solyc02g067530), SIP4H5 (Solyc01g080530), SIP4H6 (Solyc02g087490), SIP4H8 (Solyc03g033320), SIP4H9 (Solyc11 1g005200) genes in AZs of SIP4H3 RNAi line \#1, \#6 and \#7 and wild type (WT). The relative expression was calculated according to the comparative Ct method by using actin as internal standard. The asterisk indicates statistically significant differences.

SUPPLEMENTARY TABLE 1 | Table of primers used for the qPCR analysis, the subcloning of SIP4H3 CDNA in GATEWAY vector, and amplification of NPTII partial CDNA fragment. 


\section{REFERENCES}

Addicott, F. T. (1982). Abscission. (Oakland, CA: University of California Press). Alba, R., Payton, P., Fei, Z., McQuinn, R., Debbie, P., Martin, G. B., et al. (2005). Transcriptome and selected metabolite analyses reveal multiple points of ethylene control during tomato fruit development. Plant Cell 17, 2954-2965. doi: $10.1105 /$ tpc. 105.036053

Butenko, M. A., Patterson, S. E., Grini, P. E., Stenvik, G. E., Amundsen, S. S., Mandal, A., et al. (2003). INFLORESCENCE DEFICIENT IN ABSCISSION controls floral organ abscission in Arabidopsis and identifies a novel family of putative ligands in plants. Plant Cell 15, 2296-2307. doi: 10.1105/tpc.014365

Butenko, M. A., and Simon, R. (2014). Beyond the meristems: similarities in the CLV3 and IDA peptide mediated signalling pathways. J. Exp. Bot. 66, 5195-5203. doi: $10.1093 /$ jxb/erv310

Fillatti, J. J., Kiser, J., Rose, R., and Comai, L. (1987). Efficient transfer of a glyphosate tolerance gene into tomato using a binary agrobacterium tumefaciens vector. Biotechnol. 5, 726-730. doi: 10.1038/nbt0787-726

Fragkostefanakis, S., Dandachi, F., and Kalaitzis, P. (2012). Expression of arabinogalactan proteins during tomato fruit ripening and in response to mechanical wounding, hypoxia and anoxia. Plant Physiol. Biochem. 52, 112-118. doi: 10.1016/j.plaphy.2011.12.001

Fragkostefanakis, S., Kaloudas, D., and Kalaitzis, P. (2018). Pyridine 2,4-dicarboxylic acid suppresses tomato seedling growth. Front. Chem. 6:3. doi: 10.3389/ fchem.2018.00003

Fragkostefanakis, S., Sedeek, K. E., Raad, M., Zaki, M. S., and Kalaitzis, P. (2014). Virus induced gene silencing of three putative prolyl 4-hydroxylases enhances plant growth in tomato (Solanum lycopersicum). Plant Mol. Biol. 85, 459-471. doi: 10.1007/s11103-014-0197-6

Ito, Y., and Nakano, T. (2015). Development and regulation of pedicel abscission in tomato. Front. Plant Sci. 6:442. doi: 10.3389/fpls.2015.00442

Iwai, H., Tarao, A., and Satoh, S. (2013). Changes in distribution of cell wall polysaccharides in floral and fruit abscission zones during fruit development in tomato (Solanum lycopersicum). J. Plant Res. 126, 427-437. doi: 10.1007/ s10265-012-0536-0

Jiang, C. Z., Lu, F., Imsabai, W., Meir, S., and Reid, M. S. (2008). Silencing polygalacturonase expression inhibits tomato petiole abscission. J. Exp. Bot. 59, 973-979. doi: 10.1093/jxb/ern023

Kalaitzis, P., Solomos, T., and Tucker, M. L. (1997). Three different polygalacturonases are expressed in tomato leaf and flower abscission, each with a different temporal expression pattern. Plant Physiol. 113, 1303-1308. doi: 10.1104/pp.113.4.1303

Koyasu, S., Kobayashi, M., Goto, Y., Hiraoka, M., and Harada, H. (2017). Regulatory mechanisms of hypoxia-inducible factor 1 activity: two decades of knowledge. Cancer Sci. 109, 560-571. doi: 10.1111/cas.13483

Livak, K. J., and Schmittgen, T. D. (2001). Analysis of relative gene expression data using real-time quantitative PCR and the 2-11 CT Method. Methods 25, 402-408. doi: 10.1006/meth.2001.1262

Ma, C., Meir, S., Xiao, L., Tong, J., Liu, Q., Reid, M., et al. (2015). A KNOTTED1-LIKE HOMEOBOX protein regulates abscission in tomato by modulating the auxin pathway. Plant Physiol. 167, 844-853. doi: 10.1104/ pp.114.253815

Mao, L., Begum, D., Chuang, H. W., Budiman, M. A., Szymkowiak, E. J., Irish, E. E., et al. (2000). JOINTLESS is a MADS-box gene controlling tomato flower abscission zone development. Nature 406, 910-913. doi: 10.1038/35022611

Matsubayashi, Y. (2014). Posttranslationally modified small-peptide signals in plants. Annu. Rev. Plant Biol. 65, 385-413. doi: 10.1146/annurevarplant-050312-120122

Meir, S., Philosoph-Hadas, S., Sundaresan, S., Selvaraj, K. S., Burd, S., Ophir, R., et al. (2010). Microarray analysis of the abscission-related transcriptome in the tomato flower abscission zone in response to auxin depletion. Plant Physiol. 154, 1929-1956. doi: 10.1104/pp.110.160697

Nakano, T., Fujisawa, M., Shima, Y., and Ito, Y. (2014). The AP2/ERF transcription factor SlERF52 functions in flower pedicel abscission in tomato. J. Exp. Bot. 65, 3111-3119. doi: 10.1093/jxb/eru154

Nakano, T., and Ito, Y. (2013). Molecular mechanisms controlling plant organ abscission. Plant Biotechnol. 30, 209-216. doi: 10.5511/ plantbiotechnology.13.0318a
Sambrook, J., Maniatis, T., and Fritsch, E. F. (1982). Molecular cloning, a laboratory manual. (New York, NY: Cold Spring Harbor laboratory).

Semenza, G. L. (2010). Oxygen homeostasis. WIREs Syst. Biol. Med. 2, 336-361. doi: $10.1002 /$ wsbm.69

Stenvik, G. E., Butenko, M. A., Urbanowicz, B. R., Rose, J. K., and Aalen, R. B. (2006). Overexpression of INFLORESCENCE DEFICIENT IN ABSCISSION activates cell separation in vestigial abscission zones in Arabidopsis. Plant Cell 18, 1467-1476. doi: 10.1105/tpc.106.042036

Stenvik, G. E., Tandstad, N. M., Guo, Y., Shi, C. L., Kristiansen, W., Holmgren, A., et al. (2008). The EPIP peptide of INFLORESCENCE DEFICIENT IN ABSCISSION is sufficient to induce abscission in Arabidopsis through the receptor-like kinases HAESA and HAESA-LIKE 2. Plant Cell 20, 1805-1817. doi: $10.1105 /$ tpc. 108.059139

Sundaresan, S., Philosoph-Hadas, S., Riov, J., Mugasimangalam, R., Kuravadi, N. A., Kochanek, B., et al. (2016). De novo transcriptome sequencing and development of abscission zone-specific microarray as a new molecular tool for analysis of tomato organ abscission. Front. Plant Sci. 6:1258. doi: 10.3389/ fpls.2015.01258

Tabuchi, T., Ito, S., and Arai, N. (2001). Anatomical studies of the abscission process in the tomato pedicels at flowering stage. J. Jpn. Soc. Hortic. Sci. 70, 63-65. doi: $10.2503 /$ jjshs.70.63

Tranbarger, T. J., Fooyontphanich, K., Roongsattham, P., Pizot, M., Collin, M., Jantasuriyarat, C., et al. (2017). Transcriptome analysis of cell wall and NAC domain transcription factor genes during Elaeis guineensis fruit ripening: evidence for widespread conservation within monocot and eudicot lineages. Front. Plant Sci. 8:603. doi: 10.3389/ fpls.2017.00603

Tsuchiya, M., Satoh, S., and Iwai, H. (2015). Distribution of XTH, expansin, and secondary-wall-related CesA in floral and fruit abscission zones during fruit development in tomato (Solanum lycopersicum). Front. Plant Sci. 6:323. doi: $10.3389 /$ fpls.2015.00323

Tucker, M. L., and Yang, R. (2012). IDA-like gene expression in soy- bean and tomato leaf abscission and requirement for a diffusible stelar abscission signal. AoB Plants 2012:pls035. doi: 10.1093/aobpla/pls035

van Nocker, S. (2009). Development of the abscission zone. Stewart Postharvest Rev. 1, 1-6. doi: 10.2212/spr.2009.1.5

Velasquez, S. M., Marzol, E., Borassi, C., Pol-Fachin, L., Ricardi, M. M., Mangano, S., et al. (2015). Low sugar is not always good: impact of specific O-glycan defects on tip growth in Arabidopsis. Plant Physiol. 168, 808-813. doi: $10.1104 /$ pp.114.255521

Velasquez, S. M., Ricardi, M. M., Dorosz, J. G., Fernandez, P. V., Nadra, A. D., Pol-Fachin, L., et al. (2011). O-glycosylated cell wall proteins are essential in root hair growth. Science 332, 1401-1403. doi: 10.1126/ science. 1206657

Vlad, F., Spano, T., Vlad, D., Daher, F. B., Ouelhadj, A., Fragkostefanakis, S., et al. (2007). Involvement of Arabidopsis prolyl 4 hydroxylases in hypoxia, anoxia and mechanical wounding. Plant Signal. Behav. 2, 368-369. doi: $10.4161 /$ psb.2.5.4462

Wang, Z., Cao, R., Taylor, K., Briley, A., Caldwell, C., Cheng, J., et al. (2013). The properties of genome conformation and spatial gene interaction and regulation networks of normal and malignant human cell types. PLoS One 8:e58793. doi: 10.1371/journal.pone.0058793

Zhang, Y., Yang, S., Song, Y., and Wang, J. (2014). Genome-wide characterization, expression and functional analysis of CLV3/ESR gene family in tomato. BMC Genomics 15:827. doi: 10.1186/1471-2164-15-827

Conflict of Interest Statement: The authors declare that the research was conducted in the absence of any commercial or financial relationships that could be construed as a potential conflict of interest.

Copyright (® 2019 Perrakis, Bita, Arhondakis, Krokida, Mekkaoui, Denic, Blazakis, Kaloudas and Kalaitzis. This is an open-access article distributed under the terms of the Creative Commons Attribution License (CC BY). The use, distribution or reproduction in other forums is permitted, provided the original author(s) and the copyright owner(s) are credited and that the original publication in this journal is cited, in accordance with accepted academic practice. No use, distribution or reproduction is permitted which does not comply with these terms. 